\title{
FRANCIUM SPECTROSCOPY AND A POSSIBLE MEASUREMENT OF THE NUCLEAR ANAPOLE MOMENT
}

\author{
S. AUBin, E. GOMEZ, J. M. Grossman, L. A. OROZCO, M. R. PEARSON, \\ G. D. SPROUSE \\ Dept. of Physics and Astronomy, State University of New York at Stony Brook, \\ Stony Brook, NY 11794-3800, U.S.A. \\ E-mail: Luis.Orozco@sunysb.edu \\ D. P. DEMILLE \\ Dept. of Physics, Yale University, New Haven, CT 06520-8120, U.S.A.
}

The spectroscopy of francium performed in a magneto-optical trap permits quantitative comparison between $a b$ initio calculations and measurements of energy levels, electronic lifetimes, and hyperfine splittings to an accuracy comparable to that achieved in other alkali elements. This understanding is fundamental for proposed anapole moment measurements in a chain of francium isotopes.

\section{Introduction}

Francium is the heaviest of the alkali atoms and has no stable isotopes. The longest lived isotope has a half-life of 22 minutes. Since 1995 we have carried out a systematic study of the atomic and nuclear properties of Fr at the Stony Brook Superconducting LINAC in our on-line magneto-optical trap (MOT) ${ }^{1}$. Because of the large number of constituent particles in Fr, electron correlations and relativistic effects are important, but its structure remains calculable with many-body perturbation theory (MBPT). Its more than two hundred nucleons and simple atomic structure make it an attractive candidate for studies of atomic parity non-conservation and nuclear anapole moment measurements.

\section{Spectroscopy}

We have been studying the atomic spectroscopy of Fr to test the ab initio atomic theory ${ }^{2}$ that predicts a factor of 18 larger Parity Non-Conserving (PNC) effect in Fr than in Cs. Examples of our work include measurements of the lifetimes of the $7 p$ levels $^{3}$ and of the hyperfine splitting of the $7 P_{1 / 2}$ level $^{4}$ in five different isotopes ${ }^{208-212} \mathrm{Fr}$. The precision in the former (better than $0.5 \%$ ) tested MBPT calculations of the dipole matrix elements, while the precision of the latter (200 ppm) was enough to resolve the hyperfine anomaly.

Icols01 new: submitted to World Scientific on February 21, 2002 


\section{New Apparatus}

We have constructed a new apparatus for on-line magneto-optical trapping of radioactive Fr atoms at the Stony Brook LINAC for the next generation of Fr experiments. The new apparatus separates the production, trapping, and measurement regions of the experiment.

An ion beam with an energy of $\sim 100 \mathrm{MeV}$ from the accelerator strikes a target of gold or isotopically enriched platinum mounted on the end of a tungsten $\operatorname{rod}^{5}$. A heavy-ion fusion reaction $\left[{ }^{16,18} \mathrm{O}\left({ }^{197} \mathrm{Au}, x \mathrm{n}\right)\right.$ or $\left.{ }^{19} \mathrm{~F}\left({ }^{198} \mathrm{Pt}, x \mathrm{n}\right)\right]$ selectively produces the isotopes ${ }^{206-213} \mathrm{Fr}$ in the target room of the accelerator. The beam power and an auxiliary heater coil heat the target to just below its melting point, allowing the Fr to diffuse rapidly to its surface. The target ionizes the Fr as it exits. We then transport the Fr as ions to a different room for subsequent neutralization and capture in a MOT. Because the transport system is electrostatic, it is mass independent, allowing us to transport any isotope of Fr and other alkali elements. We have tested the entire apparatus from the target to the MOT with ${ }^{85} \mathrm{Rb}$. We have produced an ion beam containing in excess of $1.4 \times 10^{7} \mathrm{Fr} / \mathrm{sec}$ from the target. A small constriction separates the target and transport region from the trap, permitting differential pumping of the trap. The captured atoms can then be transferred to a second chamber for further study.

\section{Anapole moment}

The anapole moment of a nucleus is a PNC, time reversal conserving moment that arises from weak interactions between the nucleons (see the recent review by Haxton and Wieman ${ }^{6}$ ). It can only be detected in a PNC electron-nucleus interaction and reveals itself in the nucleon spin dependent part of the PNC interaction. The measurement of the Cs anapole moment by Wood et al. ${ }^{7}$, by looking at the changes in atomic PNC as a function of the hyperfine energy levels, showed that atomic PNC is a unique probe for the neutral weak interactions inside the nucleus.

The anapole moments in Fr arise from the weak interaction between the valence nucleons and the core. Flambaum, Khriplovich and Sushkov ${ }^{8}$ developed a simple model to estimate anapole moments

$$
\mathbf{a}=\frac{1}{e} \frac{G g}{\sqrt{2}} \frac{K \mathbf{I}}{I(I+1)} \kappa_{a}, \quad \kappa_{a}=\frac{9}{10} \frac{\alpha \mu}{m r_{0}} A^{2 / 3}
$$

where $G=10^{-5} \mathrm{~m}^{-2}$ is the Fermi constant of the weak interaction, and $m, I$, and $\mu$ are the mass, spin and magnetic moment of the valence nucleon. $g$ is

Icols01 new: submitted to World Scientific on February 21, 2002 
a dimensionless constant of order one describing the strength of the P-odd coupling between nucleons ${ }^{9}$. $K$ is given by $K=\left(I+\frac{1}{2}\right)(-1)^{I+\frac{1}{2}-\ell}$ and $\ell$ is the orbital angular momentum of the valence nucleon.

We have used the simple model above with the shell model parameters from our work on the hyperfine anomaly ${ }^{4}$ to estimate the anapole moments of the light $\mathrm{Fr}$ isotopes. We find $\kappa_{a}\left({ }^{211} \mathrm{Fr}\right)=0.45$, and that the anapole moment for the even-neutron $\mathrm{Fr}$ isotopes, ${ }^{209} \mathrm{Fr}$ and ${ }^{211} \mathrm{Fr}(I=9 / 2)$, will be essentially the same since both have a single unpaired nucleon, an $h_{9 / 2}$ proton.

We focus on ${ }^{211} \mathrm{Fr}$ to calculate the direct excitation between the $(7 \mathrm{~s}, \mathrm{~F}=$ $4) \rightarrow(7 s, F=5)$ hyperfine $E 1$ transition allowed by the anapole moment. We use our estimated anapole moment, the measured electric dipole values ${ }^{3}$, and first order perturbation theory (truncated at the $7 p_{1 / 2}$ states) to calculate the electric dipole matrix element between the $7 s$ hyperfine states. For example, with linear polarization transverse to the quantization axis, we calculate an expected value for the magnitude of the matrix element for $m_{F}=I-1 / 2 \rightarrow$ $m_{F}=I+1 / 2$ of $4.4 \times 10^{-12} \mathrm{ea}_{0}$ (see Ref. 9), a factor of ten larger than the similar matrix element in ${ }^{133} \mathrm{Cs}$. The ratio between the anapole induced PNC $E 1$ transition to the allowed $M 1$ hyperfine transition is $1.4 \times 10^{-9}$.

In this proposed measurement, the suggestion of Fortson ${ }^{11}$ to use an ion placed at the antinode of a standing optical wave is modified to place many trapped atoms at the antinode of a standing microwave, so the requirements of stability of the sample are relaxed. The atoms will have to be localized well within the antinode of the standing wave $(\nu \approx 50 \mathrm{GHz}, \lambda \approx 6 \mathrm{~mm})$. This approach has been suggested in the literature in the past ${ }^{12,13,14}$, and it consists of measuring the probability of transition by the direct excitation through the E1 transition (allowed mainly through the nucleon spin dependent term) between the ground hyperfine states.

The preparation of the sample of Fr will take place in the MOT. From this first trap, we will transfer the Fr to a second chamber, where the atoms will be held in a purely optical trap (dipole) at the electric antinode of a standing wave microwave cavity, so as to maximally drive the $E 1$ transition. To enhance the small probability of excitation, we will use interference with an allowed transition, such as the $M 1$, in the presence of a coordinate system with reversible handedness. Given all the constraints for preparation and transfer of the Fr atoms, we will use a Fabry-Perot configuration for the cavity, where combinations of RF and optical pulses will prepare the appropriate superposition of states. Optical interrogation, through a cycling transition, after the excitation pulses will determine how many atoms have performed the $E 1$ transition.

Recent work related to time-reversal invariance tests with traps ${ }^{15,16}$ point

Icols01 new: submitted to World Scientific on February 21, 2002 
to the many potential problems of combining traps with tests of fundamental symmetries, indicating further work is needed.

\section{Acknowledgments}

This work has been supported by the NSF. E. G. acknowledges support from CONACYT and the Fulbright-García Robles foundation.

\section{References}

1. J. E. Simsarian, A. Ghosh, G. Gwinner, L. A. Orozco G. D. Sprouse, and P. A. Voytas, Phys. Rev. Lett. 76, 3522 (1996).

2. V. A. Dzuba, V.V. Flambaum, and O. P. Sushkov, Phys. Rev. A 51, 3454 (1995).

3. J. E. Simsarian, L. A. Orozco, G. D. Sprouse, and W. Z. Zhao, Phys. Rev. A 57, 2448 (1998).

4. J. S. Grossman, L. A. Orozco, M. R. Pearson, J. E. Simsarian, G. D. Sprouse, and W. Z. Zhao, Phys. Rev. Lett. 83, 935 (1999).

5. A. R. Lipski, L. A. Orozco, M. R. Pearson, J. E. Simsarian, G. D. Sprouse, and W. Z. Zhao, Nucl. Instr. Meth. A 438, 217 (1999).

6. W. C. Haxton, C. E. Wieman, arXiv:nucl-th/0104026.

7. C. S. Wood, S. C. Bennett, D. Cho, B. P. Masterson, J. L. Roberts, C. E. Tanner, and C. E. Wieman, Science 275, 1759 (1997).

8. V. V. Flambaum, I. B. Khriplovich and O. P. Sushkov, Phys. Lett. 146B, 367 (1984).

9. I. B. Khriplovich, Parity Nonconservation in Atomic Phenomena (Gordon and Breach Science Publishers, Philadelphia, 1991).

10. After the ICOLS01 conference we received the preprint: S. G. Porsev and M. G. Koslov, arXiv:physics/0107016 with a similar calculation.

11. E. N. Fortson, Phys. Rev. Lett. 70, 2383 (1993).

12. C. E. Loving, and P. G. H. Sandars, J. Phys. B. 10, 2755 (1977).

13. V. G. Gorshkov, V. F. Ezhov, M. G. Kozlov, and A. I. Mikhailov, Sov. J. Nucl. Phys. 48, 867 (1988).

14. D. Budker in Physics Beyond the Standard Model, ed. P. Herczeg, C. M. Hoffman, and H. V. Klapdor-Klinfrothaus (World Scientific, Singapore, 1998).

15. M. V. Romalis and E. N. Fortson, Phys. Rev. A. 59, 4547 (1999).

16. C. Chin, V. Leiber, V. Vuletic, A. J. Kerman, and S. Chu, Phys. Rev. A 63, 033401 (2001). 